\title{
DEFORMATIONS OF SOME ALGEBRAIC SURFACES WITH $q=0$ AND $p_{g}=1$
}

\section{BY NAOKI HASHIMOTO}

\section{$\S 1$. Introduction.}

Let $M^{a}$ be an affine algebraic surface in $C^{3}$ defined by $h(w)=1+\sum_{\imath=1}^{3} w^{A_{\imath}}=0$ where $A_{1}, A_{2}, A_{3}$ are linearly independent non-negative integral vectors. Let $\Delta$ be the simplex in $\boldsymbol{R}^{3}$ spun by $\overrightarrow{0}, A_{1}, A_{2}$ and $A_{3}$. In $[6,7]$, Oka showed that $M^{a}$ has a canonical smooth compactification in a toric variety $W$ of dimension three. Let $A_{4}, \cdots, A_{l}$ be the other integral points on $\Delta$ and let $h_{t}(w)=h(w)$ $+\sum_{i=4}^{l} t_{i} w^{A}$. There exists a Zariski open set $U^{e}$ of $C^{l-3}$ such that the family of affine algebraic surfaces $M_{t}^{a}=\left\{h_{t}(w)=0\right\} \quad\left(t \in U^{e}\right)$ has a simultaneous smooth compactification $M_{t}$ in $W\left(M_{0}=M\right)$. This deformation is called the embedded deformation of $M$ ([7]). Let $\nu_{t}$ be the sheaf of the germs of the holomorphic section of the normal bundle of $M_{t}$ in $W$ and let $\Theta_{t}$ and $\Theta_{W}$ be the sheaves of the germ of holomorphic vector fields of $M_{t}$ and $W$ respectively. We have the canonical exact sequence:

$$
0 \longrightarrow \Theta_{t} \longrightarrow \Theta_{W} \mid M_{t} \longrightarrow \nu_{t} \longrightarrow 0 .
$$

This induces the following long exact sequence:

$$
\begin{aligned}
0 \longrightarrow H^{0}\left(M_{t}, \Theta_{t}\right) & \longrightarrow H^{0}\left(M_{t}, \Theta_{W} \mid M_{t}\right) \longrightarrow H^{0}\left(M_{t}, \nu_{t}\right) \\
& \stackrel{\delta}{\longrightarrow} H^{1}\left(M_{t}, \Theta_{t}\right) \longrightarrow H^{1}\left(M_{t}, \Theta_{W} \mid M_{t}\right) \longrightarrow H^{1}\left(M_{t}, \nu_{t}\right)
\end{aligned}
$$

In $[7]$, Oka has studied the infinitesimal displacement map

$$
\xi^{e}: T_{t} U^{e} \longrightarrow H^{0}\left(M_{t}, \nu_{t}\right),
$$

and the Kodaira-Spencer map $\delta \circ \xi^{e}$ where $\delta$ is the canonical homomorphism

$$
\delta: H^{0}\left(M_{t}, \nu_{t}\right) \longrightarrow H^{1}\left(M_{t}, \Theta_{t}\right) .
$$

The dimension of $\operatorname{Ker} \delta$ is at least 3. He gives an example (See $\S 7,[7]$ ) where $\operatorname{dim} \operatorname{Ker} \delta=3$ and $\delta$ is surjective.

Received September 14, 1987 
The purpose of this note is to give an example of an algebraic surface $M$ embedded in a toric variety $W$ such that $\operatorname{dim} \operatorname{Ker} \delta=12$ and $\delta$ is not surjective (Theorem 2.11). $\quad M$ is locally defined by $h(w)=1+w_{1}^{8}+w_{1}^{2} w_{3}^{4}+w_{1}^{3} w_{2}^{2}=0$.

The Author is greatly indebted to Professor M. Oka for many helpful suggestions and discussions, and for reading the manuscript.

\section{§ 2. Deformation of surfaces $M$.}

In this section, we study the algebraic surface $M$ introduced in $\S 1$ and its deformation $\left\{M_{t}\right\}$ through the infinitesimal displacement. We use the same notation as in $[6,7]$.

Let $M^{a}$ be the affine algebraic surface in $C^{3}$ which is defined by

$$
h(w)=1+w_{1}^{8}+w_{1}^{2} w_{3}^{4}+w_{1}^{3} w_{2}^{2}=0 .
$$

Let $\Delta$ be as in $\S 1$. $\Delta$ has 27 other integral points $A_{4}, \cdots, A_{30}$ and let $h(w, t)=$ $h(w)+\sum_{j=4}^{30} t_{j} w^{A}, \quad M_{t}^{a}$ is defined by $h(w, t)=0$. For the compactification of $M_{t}^{a}$, we consider the homogeneous polynomial $f_{\xi}(z, t)$ which is defined by

$$
f_{\varepsilon}(z, t)=h_{\Xi}\left(z_{1} / z_{0}, z_{2} / z_{0}, z_{3} / z_{0}, t\right) \cdot z_{0}^{8} .
$$

and let

$$
f(z, t)=f_{g}(z, t)+z_{2}^{L}+z_{3}^{L},
$$

for sufficiently large $L$. Let $M_{t}$ be the compactification of $M_{t}^{\alpha}$ through the troidal embedding theory as in [7]. $M_{t}$ has the following numerical invariants.

$$
K^{2}=0, \quad e\left(M_{t}\right)=24, \quad \pi_{1}\left(M_{t}\right) \cong Z / 2 Z \text { and } p_{g}=1 .
$$

Here $K$ is a canonical divisor, $e\left(M_{t}\right)$ is the topological Euler characteristic and $p_{g}$ is the geometric genus. For the calculation, we use $\S 9$ of [5]. $M_{t}$ is a minimal surface. Let us recall the compactification $M_{t}$ of $M_{t}^{a}$. Let $V_{t}=f^{-1}(0)$ ( $t$ is fixed). The dual Newton diagram $\Gamma^{*}(f)$ contains five particular vertices $P_{1}={ }^{t}(5,3,0,2), P_{2}={ }^{t}(3,5,0,2), P_{3}={ }^{t}(1,1,1,2), P_{4}={ }^{t}(1,1,2,1)$ and $P^{t}{ }^{t}(1,1,1,1)$. Let $\Sigma^{*}$ be a simplicial unimodular subdivision of $\Gamma^{*}(f)$ and let $\hat{\pi}: X \rightarrow C^{3}$ be the associated birational proper morphism and let $\tilde{V}$ be the proper transform of $V$. For each strictly positive vertex $Q$ of $\Sigma^{*}$ with $\operatorname{dim} \Delta(Q) \geqq 1$, there is a corresponding exceptional divisor $\hat{E}(Q)$ and $E(Q)$ of $\hat{\pi}: X \rightarrow C^{3}$ and $\hat{\pi}: \tilde{V} \rightarrow V$ respectively. $\hat{E}(Q)$ is a toric variety. Then it is shown in $[5,6]$ that the exceptional divisor $E(P)$ is a smooth compactification of $M_{t}^{a}$ which is a hypersurface in the toric variety $\hat{E}(P)$. We denote $\hat{E}(P)$ by $W$ hereafter. Let $\mathcal{S}$ be the 3 -simplexes of $\Sigma^{*}$ which contains $P$ as a vertex. Then $S$ gives a canonical affine coordinate system of $W$. In our case, $|\mathcal{S}|$ is 24 . For a vertex $Q$ which is adjacent to $P$ and $\operatorname{dim} \Delta(P) \cap \Delta(Q) \geqq 1$, there is a corresponding divisor $C(Q)$ of $M_{t}$. In our case, we have the divisor $C\left(T_{12}\right)$ besides $C\left(P_{\imath}\right)(i=1, \cdots, 4)$ where $T_{12}={ }^{t}(2,2,0,1)$. 
Take the following 3 -simplex $\sigma=\left(P, R, P_{2}, P_{3}\right)$ in $\mathcal{S}$ where $R=^{t}(3,4,1,3)$. $\sigma$ is fixed hereafter. The defining equation of $M_{t}$ in $C_{\sigma}^{s}$ is

$$
h_{\sigma}(y, t)=y_{1}+y_{1}^{9} y_{2}^{16}+y_{1}^{3} y_{3}^{4}+1+\sum_{j=4}^{30} t_{j} y^{B_{\jmath}}=0 \text {. }
$$

where the monomials $y^{B_{\jmath}}(j=4, \cdots, 30)$ are embedded monomials. As $l$ is 30 , the dimension of the embedded deformation is 27 . Then by Theorem (5.1) of [7], we have the next Lemma.

LEMMA 2.6.

$$
\operatorname{dim} H^{0}\left(M_{t}, \nu_{t}\right)=30 \text {. }
$$

By the Riemann-Roch theorem, we have the Euler-Poincare characteristics $\chi\left(\Theta_{t}\right)$ is -20 .

\section{LEMMA 2.7.}

$$
H^{0}\left(M_{t}, \Theta_{W} \mid M_{t}\right) \cong C^{12} \text { and } H^{0}\left(M_{t}, \Theta_{t}\right)=0 \text {. }
$$

Proof. We follow the method of calculation in $\S 7$ of [7]. Take the 3simplex $\tau=\left(P, P_{1}, S, P_{4}\right)$ in $\mathcal{S}$ where $S={ }^{t}(4,3,2,3)$. We denote $y_{\sigma \imath}, y_{\tau \imath}$ by $y_{\imath}, u_{\imath}$ respectively. Then we have $y_{1}=u_{1}^{16} u_{2}^{7} u_{3}^{-2}, y_{2}=u_{1}^{-9} u_{2}^{-4} u_{3}$ and $y_{3}=u_{1}^{-12} u_{2}^{-5} u_{3}$. Let $v \in H^{0}\left(M_{t}, \Theta_{W} \mid M_{t}\right)$. By the GAGA-principle, $v$ can be expressed in $\boldsymbol{C}_{\sigma}^{3} \cap M_{t}$ as $\sum_{\jmath=1}^{3} v_{j} \frac{\tilde{\partial}}{\partial y_{\imath}}$ where $v_{\jmath}$ is a Laurent polynomial in $y_{1}, \cdots, y_{3}$ and $\frac{\tilde{\partial}}{\partial y_{\jmath}}$ is equal to $y_{j} \frac{\partial}{\partial y_{j}}$ by definition. We may assume that $v_{j}$ has a regular form on $C\left(P_{1}\right)$ and $C\left(P_{4}\right)$ simultaneously (For the definitions of divisors $C\left(P_{2}\right)$ and regular forms, see Lemma (7.6) of [7]). Assume that the monomial $y^{\nu}$ has a non-zero coefficient in $v_{i}$. As we have

$$
y^{\nu}=u_{1}^{16 \nu_{1}-9 \nu_{2}-12 \nu_{3}} u_{2}^{7 \nu_{1}-4 \nu_{2}-5 \nu_{3}} u_{3}^{-2 \nu_{1}+\nu_{2}+\nu_{3}},
$$

we must have $8 \nu_{2}+8 \nu_{3}+8 \geqq 16 \nu_{1} \geqq 9 \nu_{2}+12 \nu_{3}-1$. Combine this with $\nu_{2} \geqq-\delta_{i 2}$, $\nu_{3} \geqq-\delta_{i 3}$ where $\delta_{i j}$ is the Kronecker's symbol. The possible cases are $\frac{\tilde{\partial}}{\partial y_{2}}$, $y_{1} y_{3} \frac{\tilde{\partial}}{\partial y_{\imath}}, \quad y_{1} y_{2} \frac{\tilde{\partial}}{\partial y_{\imath}}, \quad y_{1}^{2} y_{2} y_{3}^{2} \frac{\tilde{\partial}}{\partial y_{\imath}}, \quad y_{1}^{2} y_{2}^{2} y_{3} \frac{\tilde{\partial}}{\partial y_{\imath}}, \quad y_{1}^{2} y_{2}^{3} \frac{\tilde{\partial}}{\partial y_{\imath}}, \quad y_{1}^{3} y_{2}^{4} y_{3} \frac{\tilde{\partial}}{\partial y_{\imath}}, \quad y_{1}^{3} y_{2}^{5} \frac{\tilde{\partial}}{\partial y_{i}}$, $y_{1}^{4} y_{2}^{7} \frac{\tilde{\partial}}{\partial y_{\imath}}, \quad y_{1}^{5} y_{2}^{9} \frac{\tilde{\partial}}{\partial y_{\imath}}(i=1,2,3), y_{2}^{-1} \frac{\tilde{\partial}}{\partial y_{2}}, \quad y_{1} y_{2}^{-1} y_{3}^{2} \frac{\tilde{\partial}}{\partial y_{2}}, \quad y_{3}^{-1} \frac{\tilde{\partial}}{\partial y_{3}}, \quad y_{2} y_{3}^{-1} \frac{\tilde{\partial}}{\partial y_{3}}$, $y_{1} y_{2}^{2} y_{3}^{-1} \frac{\tilde{\partial}}{\partial y_{3}}, \quad y_{1} y_{2}^{3} y_{3}^{-1} \frac{\tilde{\partial}}{\partial y_{3}}, \quad y_{1}^{2} y_{2}^{4} y_{3}^{-1} \frac{\tilde{\partial}}{\partial y_{3}}, \quad y_{1}^{2} y_{2}^{5} y_{3}^{-1} \frac{\tilde{\partial}}{\partial y_{3}}, \quad y_{1}^{3} y_{2}^{6} y_{3}^{-1} \frac{\tilde{\partial}}{\partial y_{3}}, y_{1}^{4} y_{2}^{8} y_{3}^{-1} \frac{\tilde{\partial}}{\partial y_{3}}$, $y_{1}^{5} y_{1}^{10} y_{3} \frac{\tilde{\partial}}{\partial y_{3}}$ and $y_{1}^{6} y_{1}^{12} y_{3}^{-1} \frac{\tilde{\partial}}{\partial y_{3}}$. After checking their linear combinations in detail, we have 


$$
\begin{aligned}
v= & \alpha_{1} \frac{\tilde{\partial}}{\partial y_{1}}+\alpha_{2} y_{1} y_{3} \tilde{D}+\alpha_{3} y_{1} y_{2} \tilde{D}+\alpha_{4} y_{1}^{2} y_{2}^{2} y_{3}+\tilde{D}+\alpha_{5} y_{1}^{2} y_{2}^{3} \tilde{D} \\
& +\alpha_{6} y_{1}^{3} y_{2}^{4} y_{3} \tilde{D}+\alpha_{7} y_{1}^{3} y_{2}^{5} \tilde{D}+\alpha_{8} y_{1}^{4} y_{2}^{7} \tilde{D}+\alpha_{9} \frac{\tilde{\partial}}{\partial y_{2}}+\alpha_{10} \frac{\tilde{\partial}}{\partial y_{3}} \\
& +\alpha_{11} y_{2} y_{3}^{-1} \frac{\tilde{\partial}}{\partial y_{3}}+\alpha_{12} y_{1} y_{2}^{3} y_{3}^{-1} \frac{\tilde{\partial}}{\partial y_{3}}, \quad \text { in } \boldsymbol{C}_{\sigma} \cap M_{t},
\end{aligned}
$$

where $\alpha_{i} \in \boldsymbol{C}(i=1, \cdots, 12)$ are arbitrary constants, and

$$
\tilde{D}=2 \frac{\tilde{\partial}}{\partial y_{1}}-\frac{\tilde{\partial}}{\partial y_{2}}-\frac{\tilde{\partial}}{\partial y_{3}} .
$$

On the other hand, an easy calculation shows that $v$ as in (2.9) is holomorphic on $M_{t}$. Thus, $H^{0}\left(M_{t}, \Theta_{W} \mid M_{t}\right) \cong C^{12}$.

Now we consider $H^{0}\left(M_{t}, \Theta_{t}\right)$. Let $v$ be as in (2.9). We can write $v$ as $v=\sum_{\imath=1}^{12} \alpha_{\imath} X_{\imath}$. We show that the mapping $\theta: H^{0}\left(M_{t}, \Theta_{W} \mid M_{t}\right) \rightarrow H^{0}\left(M_{t}, \nu_{t}\right)$ is injective. Assume that $\theta(v)_{\sigma}=\sum_{i=1}^{12} \alpha_{\imath} X_{i}\left(h_{\sigma}\right) \equiv 0$ modulo $h_{\sigma}(y, t)$. We claim that all $\alpha_{\imath}(i=1, \cdots, 12)$ vanish. We have,

$$
\begin{aligned}
\sum_{i=1}^{12} \alpha_{\imath} X_{i}\left(h_{\sigma}\right) & =\sum_{\imath=1}^{12} \alpha_{\imath} X_{i}\left(h(y)+\sum_{\jmath=4}^{30} t_{\jmath} y^{A \jmath}\right) \\
& =\sum_{\jmath=1}^{30} t_{\jmath} \sum_{i=1}^{12} \alpha_{\imath} X_{i}\left(y^{A j}\right),
\end{aligned}
$$

where $t_{1}=t_{2}=t_{3}=1$. We can see that the support of $X_{i}\left(y^{A}\right)$ is included in the support of $h_{\sigma}$. As the right hand side of the above equality has no constant term, this implies $\sum_{i=1}^{12} \alpha_{\imath} X_{i}\left(h_{\sigma}\right) \neq 0$ modulo $h_{\sigma}$. This shows that the mapping $\theta$ is injective, completing the proof of Lemma (2.7).

LEMMA 2.10.

$$
H^{2}\left(M_{t}, \Theta_{W} \mid M_{t}\right)=0 .
$$

Proof. By the Serre duality, we have isomorphism

$$
\begin{aligned}
H^{2}\left(M_{t}, \Theta_{W} \mid M_{t}\right) & \cong H^{0}\left(M_{t}, \Omega_{W}^{1}(K)\right) \\
& \cong H^{0}\left(M_{t}, \Omega_{W}^{1} \mid M_{t}\left(6 C\left(P_{2}\right)-C\left(P_{3}\right)+C\left(T_{12}\right)\right)\right),
\end{aligned}
$$

as we have $K=6 C\left(P_{2}\right)-C\left(P_{3}\right)+C\left(T_{12}\right)$ by an easy calculation. $\Omega_{W}^{1}$ is the sheaf of the germs of 1 -forms. on $W$. Let $\omega=\sum_{i=1}^{3} Y_{i} d y_{i}$ be a rational 1-form and assume that the restriction of $\omega$ is in $H^{0}\left(M_{t}, \Omega_{W}^{1} \mid M_{t}\left(6 C\left(P_{2}\right)-C\left(P_{3}\right)+C\left(T_{12}\right)\right)\right.$. Let $y^{\nu}$ be a monomial with non-zero coefficient in $Y_{\imath}$ ( $i$ : fixed). Then by Lemma (7.4) of [7], we have, $\nu_{2} \geqq-6+\delta_{i 2}, \nu_{3} \geqq 1+\delta_{i 3}$ and $8 \nu_{2}+8 \nu_{3} \geqq 16 \nu_{1} \geqq 9 \nu_{2}+12 \nu_{3}$. This 
has the unique integral solution $\nu=(-2,-5,1)$. Let $\tau^{\prime}=\left(P, P_{1}, T_{12}, S^{\prime}\right)$ where $S^{\prime}={ }^{t}(5,4,1,3)$. Using $K=2 C\left(P_{2}\right)-2 C\left(P_{3}\right)+C\left(P_{4}\right)$ on $C_{\tau^{\prime}} \cap M_{t}$, and assuming that the restriction of $\omega$ is in $H^{0}\left(M_{t}, \Omega_{w}^{1}(K)\right)$, we have $\nu_{2} \geqq-2+\delta_{i 2}, \nu_{3} \geqq 2+\delta_{i 3}$, $16 \nu_{1}-9 \nu_{2}-12 \nu_{3} \geqq 0$ and $4 \nu_{1}-2 \nu_{2}-3 \nu_{3} \geqq 0$. The above integral solution does not satisfy these inequalities. Hence, we have $H^{2}\left(M_{t}, \Theta_{W} \mid M_{t}\right)=0$. This completes the proof of Lemma (2.10).

Now we are ready to show that

THEOREM 2.11. The Kodaira-Spencer map

$$
\delta \circ \xi^{e}: T_{t} U^{e} \longrightarrow H^{1}\left(M_{t}, \Theta_{t}\right),
$$

is neither injective nor surjective.

Using Theorem (5.1) of [7], we get

COROLLARY 2.12. The canonical homomorphism

$$
\delta: H^{0}\left(M_{t}, \nu_{t}\right) \longrightarrow H^{1}\left(M_{t}, \Theta_{t}\right) .
$$

is neither injective nor surjective.

Proof of Theorem 2.11. We consider the exact sequence (1.2). Considering the section $\phi \in H^{0}\left(M_{t}, \nu_{t}\right)$ such that $\phi_{\sigma}=1$, we have that the normal bundle $N_{t}$ is defined by the divisor $(\phi)=\left[16 C\left(P_{1}\right)+4 C\left(T_{12}\right)\right]$. The notation $\phi_{\sigma}$ is the same as in $\S 7,[7]$. By the Riemann-Roch theorem, we have $\chi\left(\nu_{t}\right)=30, \chi\left(\Theta_{t}\right)=-20$ and $\chi\left(\Theta_{W} \mid M_{t}\right)=10$. Then we get $H^{2}\left(M_{t}, \nu_{t}\right)=0$, and using the Lemmas (2.6), (2.7) and (2.10), $H^{1}\left(M_{t}, \nu_{t}\right)=H^{2}\left(M_{t}, \Theta_{t}\right)=0, \operatorname{dim} H^{1}\left(M_{t}, \Theta_{W} \mid M_{t}\right)=2$ and $\operatorname{dim} H^{1}\left(M_{t}, \Theta_{t}\right)$ $=20$. This completes the proof of Proposition (2.11).

Remark 2.13. Our toric variety $W$ has many "symmetries", i.e. we have $\operatorname{dim} H^{0}\left(W, \Theta_{W}\right)=12$.

We give another example of an algebraic surface $N$ in which the surjectivity of $\delta$ fails but $\operatorname{dim} \operatorname{ker} \delta=3$.

Example 2.14. Let $N^{a}$ be the affine algebraic surface in $C^{3}$ which is defined by

$$
h(w)=1+w_{2}^{5} w_{3}^{3}+w_{2}^{4} w_{3}^{4}+w_{1}^{4} w_{2}^{3} w_{3}=0 .
$$

As the homogeneous polynomial $f_{g}(z)$, we take

$$
f_{g}(z)=z_{0}^{8}+z_{2}^{5} z_{3}^{3}+z_{2}^{4} z_{3}^{4}+z_{1}^{4} z_{2}^{3} z_{3} .
$$

$N$ has the following invariants.

$$
K^{2}=0, \quad e(N)=24 \text { and } \pi_{1}(N) \cong Z / 2 Z .
$$


As $K \sim C\left(P_{1}\right)+C\left(P_{3}\right), N$ is minimal, and $p_{g}=1$. Then we have the following exact sequence :

$$
\begin{aligned}
0 & \longrightarrow H^{0}\left(N_{t}, \Theta_{W} \mid N_{t}\right) \longrightarrow H^{0}\left(N_{t}, \nu_{t}\right) \stackrel{\delta}{\longrightarrow} H^{1}\left(N_{t}, \Theta_{t}\right) \\
& \longrightarrow H^{1}\left(N_{t}, \Theta_{W} \mid N_{t}\right) \longrightarrow 0,
\end{aligned}
$$

and $\quad H^{0}\left(N_{t}, \Theta_{W} \mid N_{t}\right) \cong C^{3}, \quad \operatorname{dim} H^{0}\left(N_{t}, \nu_{t}\right)=14, \quad \operatorname{dim} H^{1}\left(N_{t}, \Theta_{t}\right)=20 \quad$ and $\operatorname{dim} H^{1}\left(N_{t}, \Theta_{W} \mid N_{t}\right)=9$. Hence we get that the Kodaira-Spencer map $\delta \circ \xi^{e}: T_{t} U^{e}$ $\rightarrow H^{1}\left(N_{t}, \Theta_{t}\right)$ is not surjective, and $\delta$ is neither injective and surjective as the case of $M$.

Remark 2.17. Minimal surfaces $M$ and $N$ with $q=0, p_{g}=1$, Euler number $=24, K^{2}=0$ and non-trivial fundamental group are classified as the minimal properly elliptic surface, and by Theorem (7.1) of p. 201, [1], the deformation of such surfaces is also minimal.

Remark 2.18. $\left\{B_{j}\right\}$ in $(2.5)$ are $(1,0,1),(1,1,0),(2,0,2),(2,1,1),(2,2,0)$, $(2,2,1),(2,3,0),(3,1,3),(3,2,2),(3,3,1),(3,4,0),(3,4,1),(3,5,0),(4,3,3)$, $(4,4,2),(4,5,1),(4,6,0),(4,7,0),(5,6,2),(5,7,1),(5,8,0),(6,8,2),(6,9,1)$, $(6,10,0),(7,11,1),(7,12,0)$ and $(8,14,0)$.

\section{REFERENCES}

[1] W. Barth, C. Peters and A. Van de Ven, Compact complex surface, Springer, Berlin-Heidelberg-New York-Tokyo, 1984.

[2] G. Kempf, F. Knudsen, D. Mumford and B. Saint-Donat, Toroidal Embeddings, Lecture Notes in Math., 339, Springer (1973).

[3] K. Kodaira, Complex Manifolds and Deformation of Complex Structures, Springer, Berlin-Heidelberg-New York, 1985.

[4] K. Kodaira and D.C. Spencer, On deformations of complex structures I, II, Annals of Math., 67 (1958), 328-466.

[5] M. OKa, On the Resolution of Hypersurface Singularities, Advanced Studies in Pure Mathematics 8 (1986), 405-436.

[6] M. OKA, Examples of Algebraic Surfaces with $q=0$ and $p_{g} \leqq 1$ which are locally Hypersurfaces, preprint.

[7] M. OKA, On the deformation of a certain type of algebraic varieties, preprint.

[8] A.N. VARCHENKo, Zeta-Function of Monodromy and Newton's Diagram, Invent. Math., 37 (1976), 253-262.

Department of Physics

Tokyo Institute of Technology

Oh-OKayama, Meguro, TOKYo, 152

JAPAN 